\title{
An Anisotropic Inverse Boundary Value Problem
}

\author{
John Sylvester* \\ Courant Institute of Mathematical Sciences \\ and \\ Mathematics Department GN-50 \\ University of Washington \\ Seattle, WA 98195
}

* Research supported by DARPA Grant \#49620-87-C0065 and NSF Grants DMS8600797 and DMS-8800153 


\begin{abstract}
We consider the impedance tomography problem for anisotropic conductivities. Given a bounded region $\Omega$ in space, a diffeomorphism $\Psi$ from $\Omega$ to itself which restricts to the identity on $\partial \Omega$, and a conductivity $\gamma$ on $\Omega$, it is easy to construct a new conductivity $\Psi_{*} \gamma$ which will produce the same voltage and current measurements on $\partial \Omega$.

We prove the converse in two dimensions (i.e. if $\gamma_{1}$ and $\gamma_{2}$ produce the same boundary measurements, then $\gamma_{1}=\Psi_{*} \gamma_{2}$ for an appropriate $\Psi)$ for conductivities which are near a constant .
\end{abstract}




\section{$\S 0$. Introduction.}

The resistance of a wire is defined by Ohm's law

$$
\delta V=I R
$$

where $\delta V$ is the potential difference across the wire and $I$ is the current flow through the wire. Both $\delta V$ and $I$ are measured quantities and $R$ is defined so that $(0.1)$ holds. The existence of the linear relationship in (0.1) is the assertion of Ohm's law. The differential version of Ohm's law is

$$
d u(x)=i(x) \rho(x)
$$

where $u(x)$ is the voltage potential at $x, d u(x)$ its differential, $i(x)$ the current flowing through $x$, and $\rho(x)$, defined by $\left(0.1^{\prime}\right)$, is called the resistivity. It will be convenient to define the conductivity $\gamma(x)$ by

$$
\gamma(x)=\frac{1}{\rho(x)}
$$

Ohm's law then has the form

$$
i(x)=\gamma(x) d u(x) .
$$

In dimensions greater than one, the current $i$ is represented by an $(n-1)$ form: the only quantity we can measure is the flow through a surface (electrode) $S$, and this quantity is given by integrating the $(n-1)$ form $i$ over the surface $S$. Ohm's law is still given by $\left(0.1^{\prime \prime}\right)$, but $\gamma(x)$ represents a mapping from differentials, or 1 -forms, $d u$, to $(n-1)$ forms $i$ : at each $x, \gamma$ maps $\wedge_{x}^{1}$, the vector space of covectors at $x$, to $\wedge_{x}^{n-1}$, the vector space of $n-1$ covectors. The existence of such a linear map $\gamma$ is again the assertion of Ohm's law.

It is customary to assume that $\gamma(x)$ is both positive definite and symmetric, that is that, for $a, b \in \wedge_{x}^{1}$

$$
\begin{gathered}
\gamma a_{\wedge} b=\gamma b_{\wedge} a \\
\gamma a_{\wedge} a=\phi(x) d x^{1} \wedge \cdots \wedge d x^{n}, \quad \phi(x) \geq \varepsilon_{\gamma}|a|^{2}>0
\end{gathered}
$$

where $x^{1}, \ldots, x^{n}$ are positively oriented euclidean coordinates and $\mid$ is the euclidean norm. If we define the $(n-1)$ forms

$$
\omega_{k}:=(-1)^{k-1} d x^{1} \wedge \cdots \wedge d x^{k-1} \wedge d x^{k+1} \wedge \cdots \wedge d x^{n}
$$


then the components $\gamma^{i j}$ of $\gamma$ are given by

$$
\gamma d x^{i}=\gamma^{i j} \omega_{j}
$$

(0.2) and (0.3) imply that the matrix $\gamma^{i j}$ is positive definite and symmetric $\left(>\varepsilon_{\gamma} I\right)$.

We shall also assume that there is no dispalcement current (i.e. no current sources or sinks within the body, which we represent by a bounded domain $\Omega$ in $\mathbb{R}^{n}$ ). Thus if $\Omega^{\prime} \subset \Omega$ is any smooth subdomain

$$
\int_{\partial \Omega^{\prime}} i=0
$$

or, using Stokes theorem

$$
\int_{\Omega^{\prime}} d i=0
$$

Since $\Omega^{\prime}$ is arbitrary, we conclude that

$$
d i=0 \text { in } \Omega
$$

or, substituting $\left(0.1^{\prime \prime}\right)$

$$
d \gamma d u=0 \text { in } \Omega
$$

which is, in coordinates

$$
\frac{\partial}{\partial x_{i}} \gamma^{i j} \frac{\partial}{\partial x^{j}} u=0
$$

The conductivities we have discussed so far are anisotropic. A conductivity is called isotropic if the relationship between voltage and current is independent of direction. That is, if we first measure the current due to a voltage potential, and then rotate the voltage potential, the current we measure will be the rotation of the first measurements. In our framework, the euclidean rotation group, $\mathcal{O}(n)$, acts naturally on both $\wedge_{x}^{1}$ and $\wedge_{x}^{n-1}$ so that, for any $o \in \mathcal{O}(n)$, the composite map

$$
\wedge_{x}^{1} \stackrel{o^{-1} \gamma(x) o}{\rightarrow} \wedge_{x}^{n-1}
$$

makes sense. $\gamma$ is isotropic at $x$ if

$$
\gamma(x)=o^{-1} \gamma(x) o \quad \forall o \in \mathcal{O}(n)
$$

One isotropic conductivity is the euclidean conductivity $e$, the conductivity whose component matrix, $e^{i j}$ equals the identity in euclidean coordinates. 
In this paper we shall study the impedance tomography problem: we wish to infer information about the conductivity $\gamma(x)$ inside a region $\Omega$ from voltage and current measurements at the boundary. The set of boundary measurements may be described as

$$
\mathcal{M}_{\gamma}=\left\{(f, \omega)|f=u|_{\partial \Omega}, \omega=\left.\gamma d u\right|_{\partial \Omega} ; u \text { solves }(0.6)\right\}
$$

$\mathcal{M}_{\gamma}$ is a subspace of $C^{\infty}(\partial \Omega) \times C^{\infty}\left(\wedge^{n-1}(\partial \Omega)\right)$; it is in fact, the graph of the linear operator

$$
C^{\infty}(\partial \Omega) \stackrel{\Lambda_{\gamma}}{\rightarrow} C^{\infty}\left(\wedge^{n-1}(\partial \Omega)\right)
$$

where

$$
\Lambda_{\gamma} f:=\left.\gamma d u\right|_{\partial \Omega}
$$

and $u$ is the unique solution to (0.6) such that

$$
\left.u\right|_{\partial \Omega}=f
$$

We can formulate the impedance tomography problem as follows: how much information about $\gamma$ can be detected from knowledge of the mapping $\Lambda_{\gamma}$ ? Much is known in the case that $\gamma$ is assumed to be isotropic.

For an isotropic conductivity which is a priori known to be real analytic [KV I], piecewise real analytic [KVIII], or smooth $\left(C^{3}\right)$ and sufficiently close to a constant [SU I], knowledge of $\Lambda_{\gamma}$ suffices to determine $\gamma$ uniquely. In dimension three and higher, in fact, any smooth $\left(C^{2}\right) \gamma$ is uniquely determined by $\Lambda_{\gamma}[\mathrm{SU} \mathrm{II}]$. The $C^{2}$ hypothesis can even be relaxed $([\mathrm{I}])$.

For an anisotropic conductivity, however, it has been observed [KV II] that this is not the case. Any diffeomorphism of $\Omega$ which fixes the boundary can be used to construct a new conductivity with the same voltage to current map. In this paper we show that in dimension two, and under the a priori hypothesis that the determinant of $\gamma$ is $\left(C^{3}\right)$ close to constant, the converse is true. If $\gamma_{1}$ and $\gamma_{2}$ have the same voltage to current map, then there exists a diffeomorphism which fixes the boundary and transforms $\gamma_{1}$ to $\gamma_{2}$. A result of this nature for real analytic conductivities (in dimensions $\geq 3$ ) appears in [LU].

In $\S 1$, we shall state the theorem precisely and prove it, based on three propositions. $\S 2$ and $\S 3$ are devoted to the proofs of these propositions.

The author would like to thank Bob Kohn, who made substantial contributions to this work; and also F. H. Lin who proved a version of theorem 1.0 under the additional hypothesis, $\operatorname{det} \gamma \equiv 1$. Thanks are also due to Percy Deift, Jack Lee, Gunther Uhlmann, and Tom Wolff for many helpful conversations. 
$\S 1$.

Let $\Psi$ denote a diffeomorphism from a smooth bounded domain $\Omega \subset \mathbb{R}^{n}$ to itself. We may define the push forward, $\Psi_{*} \gamma$ of the conductivity $\gamma$

$$
\gamma: \wedge^{1}(\Omega) \rightarrow \wedge^{n-1}(\Omega)
$$

to be

$$
\left(\Psi_{*} \gamma\right) \alpha=\Psi_{*}\left(\gamma\left(\Psi^{*} \alpha\right)\right)
$$

where $\Psi^{*} \alpha$ denotes the pull back of the 1 -form $\alpha$ and $\Psi_{*}=\left(\Psi^{-1}\right)^{*}$ denotes the pull back by $\Psi^{-1}$ acting on the $n-1$ form $\gamma\left(\Psi^{*} \alpha\right)$. In coordinates, (1.0) reads

$$
\left(\Psi_{*} \gamma(y)\right)^{\ell m}=\frac{\frac{\partial \Psi^{\ell}}{\partial x^{i}} \gamma^{i j} \frac{\partial \Psi^{m}}{\partial x^{j}}}{\operatorname{det}\left(\frac{\partial \Psi}{\partial x}\right)} \circ \Psi^{-1}(y) .
$$

Note that if $u$ satisfies

$$
d \gamma d u=0
$$

then $\Psi_{*} u=u \circ \Psi^{-1}$ satisfies

$$
\begin{aligned}
d \Psi_{*} \gamma d\left(\Psi_{*} u\right) & =d \Psi_{*}\left(\gamma\left(\Psi^{*} d \Psi_{*} u\right)\right) \\
& =\Psi_{*} d \gamma d u \\
d \Psi_{*} \gamma d\left(\Psi_{*} u\right) & =0 .
\end{aligned}
$$

If $\psi$ is a smooth diffeomorphism of $\partial \Omega$ we may form the push forward $\psi_{*} \Lambda_{\gamma}$ of the voltage to current map (recall (0.7))

$$
C^{\infty}(\partial \Omega) \stackrel{\Lambda_{\gamma}}{\rightarrow} \wedge^{n-1}(\partial \Omega)
$$

by

$$
\left(\psi_{*} \Lambda_{\gamma}\right) f=\psi_{*} \Lambda_{\gamma}\left(\psi^{*} f\right) .
$$

If we choose

$$
\psi=\left.\Psi\right|_{\partial \Omega},
$$

the unique solution to the Dirichlet problem

$$
\begin{gathered}
d\left(\Psi_{*} \gamma\right) d v=0 \\
\left.v\right|_{\partial \Omega}=f \circ \psi^{-1},
\end{gathered}
$$


is just $v=u \circ \Psi^{-1}$ where

$$
\begin{gathered}
d \gamma d u=0 \\
\left.u\right|_{\partial \Omega}=f .
\end{gathered}
$$

This implies the equality of operators

$$
\Lambda_{\Psi_{*} \gamma}=\psi_{*} \Lambda_{\gamma} \quad \text { when } \psi=\left.\Psi\right|_{\partial \Omega}
$$

If, in (1.2), we take $\psi$ to be the identity we see that

$$
\Lambda_{\Psi_{*} \gamma}=\Lambda_{\gamma}
$$

Thus, given any $\gamma$ and any diffeomorphism $\Psi$ which fixes the boundary, we can construct a (generally) different conductivity, $\Psi_{*} \gamma$, with the same voltage to current map. Our theorem is a partial converse in dimension two. Our hypotheses shall make reference to the norm of $\gamma$ in the $C^{3}$ topology; by this we mean

$$
\|\gamma\|_{C^{3}}=\sup _{\substack{x \in \bar{\Omega} \\ i, j=1,2 \\|\alpha| \leq 3}}\left|D^{\alpha} \gamma^{i j}\right|
$$

where the $\gamma^{i j}$ and all derivatives are computed in euclidean coordinates. We shall assume that the ellipticity constant, $\varepsilon_{\gamma}$ (recall $(0.3)$ ), is equal to one; this can be accomplished by multiplying $\gamma$ by a constant.

Theorem 1.0. Let $\Omega$ be a bounded domain in $\mathbb{R}^{2}$ with $C^{3}$ boundary and let $\gamma_{1}$ and $\gamma_{2}$ be $C^{3}$ conductivities with

$$
\left\|\gamma_{\ell}\right\|_{C^{3}} \leq M \text { for } \ell=1,2 \quad ; \quad \varepsilon_{\gamma}=1
$$

There exists $\varepsilon=\varepsilon(\Omega, M)$ such that if

$$
\left\|\log \left(\operatorname{det} \gamma_{\ell}\right)\right\|_{C^{3}}<\varepsilon \text { for } \ell=1,2
$$

and

$$
\Lambda_{\gamma_{1}}=\Lambda_{\gamma_{2}}
$$

then there exists a $C^{3}$ diffeomorphism $\Psi$ with

$$
\gamma_{1}=\Psi_{*} \gamma_{2} \quad ;\left.\quad \Psi\right|_{\partial \Omega}=I
$$


Remark 1.0 We could replace hypothesis (1.4) in the theorem by

$$
\gamma_{\ell} \text { is real analytic for } \ell=1,2 \text {. }
$$

This would require verifying real analyticity in propositions 1.1 and 1.3 below and replacing proposition 1.2 with the analagous theorem for real analytic conductivities, which was proved in $[\mathrm{KV} \mathrm{I}]$ in the isotropic case.

Theorem 1.0 will be a direct consequence of the following two propositions:

Proposition 1.1. Let $\Omega$ be a bounded domain in $\mathbb{R}^{2}$ with $C^{3}$ boundary, let $\gamma_{1}$ and $\gamma_{2}$ be $C^{3}$ conductivities on $\bar{\Omega}$, and let $\phi$ be a $C^{3}$ diffeomorphism of $\partial \Omega$ such that

$$
\phi_{*} \Lambda_{\gamma_{1}}=\Lambda_{\gamma_{2}}
$$

then $\phi$ extends to a $\gamma_{1}-\gamma_{2}$ conformal diffeomorphism of $\bar{\Omega}$. That is, there exists a diffeomorphism $\Phi$ mapping $\bar{\Omega}$ to itself such that

$$
\Phi_{*} \gamma_{1}=\left(\frac{\operatorname{det} \gamma_{1} \circ \Phi^{-1}}{\operatorname{det} \gamma_{2}}\right)^{1 / 2} \gamma_{2}
$$

Furthermore,

$$
\|\Phi\|_{C^{3}} \leq K=K\left(\left\|\gamma_{1}\right\|_{C^{3}},\left\|\gamma_{2}\right\|_{C^{3}},\|\phi\|_{C^{3}}, \Omega\right)
$$

Proposition 1.2. Let $\Omega$ be a bounded domain in $\mathbb{R}^{2}$ with $C^{3}$ boundary and let $\gamma$ be a $C^{3}$ conductivity on $\bar{\Omega}$. There exists $\varepsilon=\varepsilon\left(\|\gamma\|_{C^{3}}, \Omega\right)$ such that, if $\beta$ and $\operatorname{det} \gamma$ satisfy

$$
\|\log \beta\|_{C^{3}}+\|\log (\operatorname{det} \gamma)\|_{C^{3}} \leq \varepsilon
$$

and

$$
\Lambda_{\gamma}=\Lambda_{\beta \gamma}
$$

then

$$
\beta \equiv 1
$$

Proposition 1.1 is the main technical result in the paper, and will be proved in $\S 3$. In the case that $\gamma$ is isotropic, proposition 1.2 is just a restatement of the main theorem in [SU I]. The existence of isothermal coordinates will allow us to reduce to that case. In fact, we shall need a precise version of isothermal coordinates to prove proposition 1.1. To this end, we shall prove in $\S 2$ 
Proposition 1.3. Let $\Omega$ be a bounded domain in $\mathbb{R}^{2}$ with $C^{3}$ boundary and let $\gamma$ be a $C^{3}$ conductivity on $\bar{\Omega}$, then there exists a $C^{3}$ domain $\Omega^{\prime}$ and a $\gamma$-e conformal diffeomorphism $\Phi$ from $\Omega$ to $\Omega^{\prime}$; that is, there exists a $\Phi$ such that

$$
\Phi_{*} \gamma=\left(\operatorname{det} \gamma \circ \Phi^{-1}\right) e
$$

Furthermore,

$$
\|\Phi\|_{C^{3}}+\left\|\Phi^{-1}\right\|_{C^{3}} \leq K=K\left(\Omega,\|\gamma\|_{C^{3}}\right)
$$

Proof of Proposition 1.2 Let $\Phi$ be as in proposition 1.3 and let $\phi$ denote $\left.\Phi\right|_{\partial \Omega}$; then (1.11) implies

$$
\phi_{*} \Lambda_{\gamma}=\phi_{*} \Lambda_{\beta \gamma}
$$

which yields, according to (1.2),

$$
\Lambda_{\Phi_{*} \gamma}=\Lambda_{\Phi_{*} \beta \gamma}
$$

This gives, according to (1.12),

$$
\Lambda_{\left(\operatorname{det} \gamma \circ \Phi^{-1}\right) e}=\Lambda_{\left((\beta \operatorname{det} \gamma) \circ \Phi^{-1}\right) e}
$$

According to theorem 0.2 of [SU I], if (1.14) holds and

$$
\left\|\log (\beta \operatorname{det} \gamma) \circ \Phi^{-1}\right\|_{C^{3}}+\left\|\log (\operatorname{det} \gamma) \circ \Phi^{-1}\right\|_{C^{3}} \leq \delta=\delta\left(\Omega^{\prime}\right)
$$

then we may conclude that

$$
\operatorname{det} \gamma \circ \Phi^{-1}=\beta \operatorname{det} \gamma \circ \Phi^{-1}
$$

or

$$
\beta \equiv 1
$$

To guarantee (1.15), we need insist that $\varepsilon$ in (1.10) satisfies

$$
\varepsilon(1+\varepsilon)\left\|\Phi^{-1}\right\|_{C^{3}} \leq \delta
$$

or

$$
\varepsilon \leq \frac{\delta}{2 K}
$$

where $\mathrm{K}$ is the constant which appears in (1.13). 
Proof of Theorem 1.0 As a consequence of (1.5), (1.7) holds with $\phi$ equal to the identity; proposition 1.1 implies the existence of $\Phi$ extending $\phi$ such that

$$
\begin{aligned}
\Phi_{*} \gamma_{1} & =\left(\frac{\operatorname{det} \gamma_{1} \circ \Phi^{-1}}{\operatorname{det} \gamma_{2}}\right)^{1 / 2} \gamma_{2} \\
& =\beta \gamma_{2}
\end{aligned}
$$

Therefore

$$
\begin{aligned}
\Lambda_{\beta \gamma_{2}} & =\Lambda_{\Phi_{*} \gamma_{1}} \\
& =\phi_{*} \Lambda_{\gamma_{1}}=\Lambda_{\gamma_{1}}=\Lambda_{\gamma_{2}}
\end{aligned}
$$

as $\phi$ is the identity map.

We may now apply proposition 1.2 [ (1.3),(1.4), and (1.9) imply (1.10)] to conclude that $\beta \equiv 1$, or

$$
\Phi_{*} \gamma_{1}=\gamma_{2}
$$

In the remainder of this section we give a brief discussion of proposition 1.1; its proof will appear in $\S 3$. We begin by stating and proving a special case of proposition 1.1, which illustrates the proof of the theorem in a particularly simple setting. We take $\mathbf{D}$ to be the unit disk in $\mathbb{R}^{2}$, we have

Proposition 1.1'. Let $\phi$ be a $C^{2}$ diffeomorphism of the circle $(\partial \mathbf{D})$ such that

$$
\phi_{*} \Lambda_{e}=\Lambda_{e} ;
$$

then $\phi$ extends to a conformal map of $\mathbf{D}$ to itself.

Proof. We shall extend $\phi$ to a conformal map by solving

$$
\begin{aligned}
\bar{\partial} v & =0 \text { in } \mathbf{D} \\
\left.v\right|_{\partial D} & =e^{i \phi} .
\end{aligned}
$$

If we make use of the projection operators

$$
\begin{gathered}
P_{+} f=\sum_{n=0}^{\infty} f_{n} e^{i n \theta} ; f_{n}=\frac{1}{2 \pi} \int_{0}^{2 \pi} f(\theta) e^{-i n \theta} d \theta \\
P_{-} f=\sum_{n=-1}^{-\infty} f_{n} e^{i n \theta},
\end{gathered}
$$


then (1.17) has a solution exactly when

$$
P_{-}\left(e^{i \phi}\right)=0
$$

Now, on D,

$$
\Lambda_{e} f=\left(\sum_{n=-\infty}^{\infty}|n| e^{i n \theta} f_{n}\right) d \theta
$$

or

$$
\Lambda_{e} f=\left(P_{+}\left(\frac{\partial f}{\partial \theta}\right)-P_{-}\left(\frac{\partial f}{\partial \theta}\right)\right) \frac{d \theta}{i} .
$$

(1.16) implies that

$$
\Lambda_{e}(f \circ \phi)=\phi^{*}\left(\Lambda_{e} f\right)
$$

If we choose

$$
f(\theta)=e^{i \theta}
$$

and use (1.20), this becomes

$$
\left(P_{+}\left(\frac{\partial e^{i \phi}}{\partial \theta}\right)-P_{-}\left(\frac{\partial e^{i \phi}}{\partial \theta}\right)\right) d \theta=\left(\frac{\partial e^{i \phi}}{\partial \theta}\right) d \theta
$$

from which we conclude that

$$
P_{-}\left(\frac{\partial}{\partial \theta}\left(e^{i \phi}\right)\right)=0
$$

so that

$$
P_{-} e^{i \phi}=\text { constant }
$$

But the constant must be zero (see (1.18)). Hence

$$
P_{-}\left(e^{i \phi}\right)=0
$$

and we have verified (1.19); so that (1.17) has a unique solution. The fact that $v$ is a diffeomorphism follows from the argument principle and the fact that $\phi$ is $1-1$ and onto.

In the proof of proposition 1.1', we showed that we could solve the Cauchy problem (1.17) if the hypothesis (1.16) was satisfied. Proposition 1.1 can also be viewed as the statement that, in the presence of hypothesis (1.7) we can solve a certain Cauchy problem for a first order, two by two, linear elliptic system. We seek $\Phi$ such that

$$
\Phi_{*} \gamma_{1}=\beta \gamma_{2}
$$


where $\beta$ is a scalar function. In $\mathbb{R}^{2}$,

$$
\gamma: \wedge^{1} \rightarrow \wedge^{1}
$$

so that the determinant of $\gamma$ is a well defined function. Hence, if we find $\Phi$ satisfying (1.21)

$$
\begin{aligned}
\operatorname{det} \Phi_{*} \gamma_{1} & =\operatorname{det} \gamma_{1} \circ \Phi^{-1} \\
& =\beta^{2} \operatorname{det} \gamma_{2} .
\end{aligned}
$$

$\beta$ must be positive so that (0.3) is satisfied, so that

$$
\beta=\left(\frac{\operatorname{det} \gamma_{1} \circ \Phi^{-1}}{\operatorname{det} \gamma_{2}}\right)^{1 / 2}
$$

To solve (1.21), let $\omega$ and $\theta$ be $\gamma_{2}$ orthonormal 1-forms; i.e.

$$
\begin{aligned}
& \omega_{\wedge} \gamma_{2} \theta=0 \\
& \omega_{\wedge} \gamma_{2} \omega=\theta_{\wedge} \gamma_{2} \theta=1 .
\end{aligned}
$$

If (1.21) holds, then

$$
\begin{aligned}
\omega_{\wedge}\left(\Phi_{*} \gamma_{1}\right) \theta & =0 \\
\omega_{\wedge}\left(\Phi_{*} \gamma_{1}\right) \omega & =\theta_{\wedge}\left(\Phi_{*} \gamma_{1}\right) \theta
\end{aligned}
$$

or equivalently,

$$
\begin{aligned}
\Phi^{*} \omega_{\wedge} \gamma_{1}\left(\Phi^{*} \theta\right) & =0 \\
\Phi^{*} \omega_{\wedge} \gamma_{1}\left(\Phi^{*} \omega\right) & =\Phi^{*} \theta_{\wedge} \gamma_{1} \Phi^{*} \theta .
\end{aligned}
$$

Now (1.24) can only be satisfied if, for some scalar valued function $a$,

$$
\Phi^{*} \omega=a \gamma_{1}\left(\Phi^{*} \theta\right) .
$$

Inserting (1.26) into (1.25) yields

$$
a^{2}=\left(\operatorname{det} \gamma_{1}\right)^{-1} .
$$

To maintain (0.3) we must choose $a$ to be positive; hence the nonlinear system (1.24), (1.25) can be replaced by (it is straightforward to check the equivalence) the linear elliptic first order system

$$
\Phi^{*} \omega=\frac{\gamma_{1}}{\left(\operatorname{det} \gamma_{1}\right)^{1 / 2}} \Phi^{*} \theta
$$

Equation (1.27) is independent of the choice of $\theta$ and $\omega$ satisfying (1.22) and (1.23). The ellipticity of the system can easily be verified directly. It is perhaps easier to note that, if $\Psi_{1}$ and $\Psi_{2}$ are isothermal coordinates (whose existence is guaranteed by proposition 1.3) for $\gamma_{1}$ and $\gamma_{2}$ respectively, then $\tilde{\Phi}=\Psi_{1}^{-1} \circ \Phi \circ \Psi_{2}$ satisfies the Cauchy Riemann equations.

Proposition 1.1 states that we can solve the Cauchy problem for (1.27) when (1.7) holds. In fact, a direct corollary of proposition 1.1 is 
Corollary 1.4. There exists a solution to (1.27) with

$$
\left.\Phi\right|_{\partial \Omega}=\phi
$$

if and only if

$$
\phi_{*} \Lambda_{\gamma_{1}}=\Lambda_{\beta \gamma_{2}}
$$

for some scalar valued function $\beta$.

Proof The necessity follows from (1.2) and the sufficiency from proposition 1.1 with $\gamma_{2}$ replaced by $\beta \gamma_{2}$. 
$\S 2$.

In this section we prove proposition 1.3, as well as develop some special solutions to the differential equation (0.6) which will be essential to the proof of proposition 1.1. We shall make use of the weighted (at infinity) $L^{p}$ spaces, $L_{\delta}^{p}$, which are the completions of $C_{0}^{\infty}\left(\mathbb{R}^{2}\right)$ in the norms

$$
\|u\|_{p, \delta}=\left\|\left(1+|x|^{2}\right)^{\delta / 2} u\right\|_{p} \quad ; \quad \delta \in \mathbb{R}
$$

$L_{\delta}^{p}$ are complex interpolation spaces; in particular, if $T$ is a linear map

$$
L_{\delta_{i}}^{p_{i}} \stackrel{T}{\rightarrow} L_{\varepsilon_{i}}^{q_{i}} ; i=0,1
$$

with bounds $\|T\|_{i}$, then $T$ is bounded from

$$
L_{\delta(s)}^{p(s)} \stackrel{T}{\rightarrow} L_{\varepsilon(s)}^{q(s)}
$$

where

$$
\begin{aligned}
& 0<s<1 \\
\frac{1}{p(s)}= & (1-s) \frac{1}{p_{0}}+s \frac{1}{p_{1}} \\
\frac{1}{q(s)}= & (1-s) \frac{1}{q_{0}}+s \frac{1}{q_{1}} \\
\delta(s)= & (1-s) \delta_{0}+s \delta_{1} \\
\varepsilon(s)= & (1-s) \varepsilon_{0}+s \varepsilon_{1} .
\end{aligned}
$$

Furthermore,

$$
\|T\|_{s} \leq\|T\|_{0}^{1-s}\|T\|_{1}^{s}
$$

For the general approach to complex interpolation see $[\mathrm{S}]$; in this instance, the relevant analytic function is, for $a \in L_{\delta}^{p}$,

$$
f(z)=\left(1+|x|^{2}\right)^{1 / 2\left(\frac{1-z}{p_{0}}+\frac{z}{p_{1}}\right) p \delta-(1-z) \delta_{0}-z \delta_{1}} a^{p\left(\frac{1-z}{p_{0}}+\frac{z}{p_{1}}\right)}
$$

We shall use the notation

$$
\begin{aligned}
& \bar{\partial}=\frac{1}{2}\left(\frac{\partial}{\partial x}+i \frac{\partial}{\partial y}\right) \\
& \partial=\frac{1}{2}\left(\frac{\partial}{\partial x}-i \frac{\partial}{\partial y}\right) .
\end{aligned}
$$

We now state and prove the precise version of the existence of isothermal coordinates which we will need below. The letter $e$ will be used to denote the euclidean conductivity. 
Proposition 2.1. Suppose that

$$
\gamma \in C^{1,1}\left(\mathbb{R}^{2}\right)\left(\text { resp. } C^{3}\left(\mathbb{R}^{2}\right)\right)
$$

and

$$
\gamma=e \text { for }|x|>R
$$

then there exists $\beta>0$ such that, for all $(p, \delta)$ satisfying $|p-2|+|\delta+1|<\beta,-\frac{2}{p}<\delta<1-\frac{2}{p}$ there exists a unique $f \in L_{\delta}^{p}$ such that $F=z+f$ is a $C^{2}$ (resp. $\left.C^{3}\right)$ diffeomorphism from $\mathbb{R}^{2}$ to itself satisfying

$$
F_{*} \gamma=\left(\operatorname{det} \gamma \circ F^{-1}\right)^{1 / 2} e
$$

In addition,

$$
\begin{gathered}
\left\|D^{\alpha} f\right\|_{L_{\delta+1}^{p}} \leq K\left(p, \delta, M, R,\|\gamma\|_{C^{1,1}}\right) \quad \text { for } \quad 0 \leq|\alpha| \leq 3 \\
\left\|D^{\alpha} f\right\|_{L_{\delta+1}^{p}} \leq K\left(p, \delta, M, R,\|\gamma\|_{C^{3}}\right) \quad \text { for } \quad 0 \leq|\alpha| \leq 4 .
\end{gathered}
$$

Furthermore,

$$
F^{-1}=z+h
$$

with $h$ satisfying (2.5) and (2.6).

This proposition is a modification of the proof of the existence of isothermal coordinates given by Ahlfors in [A]. The function spaces we need are somewhat different, and we choose some different normalizations, but our approach parallels his. Instead of Riemanian metrics we are dealing with conductivities (which we may identify with (co)metrics tensor n-forms), however, from the conformal point of view they may be treated similiarly.

In order to be consistent with the notation used in $[\mathrm{A}]$, we shall, in this section, use only euclidean coordinates, and in lemma 2.2 below use $\gamma$ to denote the positive definite matrix of coefficients defined in (0.5) with these coordinates.

The possible point of confusion is the following: $\gamma$ maps 1-forms to (n-1)-forms; in order to define the $\gamma^{i j}$ 's we must choose two bases, as was done in (0.4) and (0.5). However, in two dimensions $\gamma$ maps 1-forms to 1-forms, but the bases we chose in (0.4) and (0.5) are different. Hence, although the trace and determinant of the mapping are well defined 
functions in two dimensions, they need not be the trace and determinant of the matrix of $\gamma^{i j}$ 's (it turns out that the determinant of the mapping actually equals the determinant of the $\gamma^{i j}$ 's and the trace of the mapping is always zero).

The proof of proposition 2.1 will follow from a series of lemmas. We use euclidean coordinates throughout. In lemma 2.2 below, (2.8) is exactly (1.27) in the special case that $\gamma_{2}$ is (conformal to) the euclidean conductivity.

Lemma 2.2. (2.4) holds if and only if

$$
\bar{\partial} F=\mu_{\gamma} \partial F \quad ; \quad \mu_{\gamma}=\frac{\gamma^{11}-\gamma^{22}+2 i \gamma^{12}}{\gamma^{11}+\gamma^{22}+2 \sqrt{\operatorname{det} \gamma}}
$$

Proof. If we write $F=F_{1}+i F_{2}$ and abuse notation by writing $\gamma$ for the matrix of $\gamma^{i j}$ 's from (0.5) and $d F$ for the column vector $\left(\frac{\partial F}{\partial x^{1}}, \frac{\partial F}{\partial x^{2}}\right)^{T},(2.4)$ becomes

$$
\begin{gathered}
d F_{1}^{T} \gamma d F_{1}=d F_{2}^{T} \gamma d F_{2} \\
d F_{1}^{T} \gamma d F_{2}=0 .
\end{gathered}
$$

Now (2.10) implies that

$$
d F_{1}=\lambda\left(\begin{array}{cc}
0 & -1 \\
1 & 0
\end{array}\right) \gamma d F_{2}
$$

where $\lambda$ is a scalar. Inserting (2.11) into (2.9) yields $\lambda= \pm(\operatorname{det} \gamma)^{1 / 2}$ and we must choose $\lambda$ positive so that $F_{*} \gamma$ is a positive definite matrix. Inserting this into (2.11) yields a linear system which can be seen to be equivalent to (2.8) (see [CH] page 351 ).

We shall now discuss (2.8). We note that

$$
\left|\mu_{\gamma}\right|^{2}=\frac{\left(\gamma^{11}+\gamma^{22}\right)^{2}-4 \operatorname{det} \gamma}{\left(\gamma^{11}+\gamma^{22}+2 \sqrt{\operatorname{det} \gamma}\right)^{2}}
$$

so that for

$$
\begin{gathered}
I<\gamma<M I \\
\left|\mu_{\gamma}\right| \leq 1-\varepsilon ; \varepsilon=\varepsilon(M)>0
\end{gathered}
$$


We shall need

Lemma 2.3. Let

$$
1<p<\infty, \quad-\frac{2}{p}<\delta<1-\frac{2}{p}
$$

There exists a constant $K(p, \delta)$ and a unique $L_{\delta}^{p}$ solution to

$$
\bar{\partial} u=v \in L_{\delta+1}^{p}
$$

Furthermore,

$$
\begin{aligned}
& \|u\|_{p, \delta}+\|D u\|_{p, \delta+1} \leq K\|v\|_{p, \delta+1} \\
& |u(z)| \leq K\|v\|_{p, \delta+1}|z|^{-2 / p-\delta} \quad \text { for } p>2 .
\end{aligned}
$$

Proof. We refer to Theorem 2.1 of [NW] (page 275) for (2.15). To summarize, one first shows that the only $L_{\delta}^{p}$ solution is the one given by

$$
u(z)=\int_{\mathbb{R}^{2}} \frac{v(w)}{z-w} \frac{d w_{\wedge} d \bar{w}}{2 \pi i}
$$

and then applies Hölder's inequality to estimate $\|u\|_{p, \delta}$ and a Calderon-Zygmund type lemma to estimate $\|D u\|_{p, \delta}$. The inequality (2.16) is also just Hölder's inequality applied to $(2.17)$, namely

$$
\begin{aligned}
& |u(z)| \leq\left(\int\left(\frac{1}{|z-w|(1+|w|)^{\delta+1}}\right)^{q} d w\right)^{1 / q}\|v\|_{p, \delta+1} \\
& \leq\left(\int_{\mathbb{R}^{2}}\left(\frac{1}{|1-x||x|^{\delta+1}}\right)^{q} d x\right)^{1 / q}|z|^{-2 / p-\delta}\|v\|_{p, \delta+1} .
\end{aligned}
$$

where $\frac{1}{q}=1-\frac{1}{p}$. The conditions $(p>2)$ and (2.14) imply that the integral is finite and that $-2 / p-\delta<0$.

Lemma 2.4. There exists $\beta>0$ such that for all $(p, \delta)$ satisfying

$$
\begin{array}{r}
\left|\frac{1}{p}-\frac{1}{2}\right|+|\delta+1|<\beta \\
-\frac{2}{p}<\delta<1-\frac{2}{p}
\end{array}
$$


then both of the mappings

$$
\left(I-\mu_{\gamma} \partial(\bar{\partial})^{-1}\right): L_{\delta+1}^{p} \rightarrow L_{\delta+1}^{p}
$$

and

$$
\left(I-\mu_{\gamma} \bar{\partial}\left(\partial^{-1}\right)\right): L_{\delta+1}^{p} \rightarrow L_{\delta+1}^{p}
$$

are invertible and satisfy

$$
\left\|\left(I-\mu_{\gamma} \bar{\partial}\left(\partial^{-1}\right)\right)^{-1}\right\|,\left\|\left(I-\mu_{\gamma} \partial(\bar{\partial})^{-1}\right)^{-1}\right\| \leq \frac{2}{\varepsilon}
$$

where $\varepsilon$ is defined in (2.13).

Proof. We shall treat only the first operator, the second is analagous.

$$
\partial(\bar{\partial})^{-1}: L_{0}^{2} \rightarrow L_{0}^{2}
$$

is bounded with norm 1. It follows from (2.15) that

$$
\partial(\bar{\partial})^{-1}: L_{\beta+1}^{q} \rightarrow L_{\beta+1}^{q}
$$

is bounded with norm $M=M(q, \beta)$.

By interpolation,

$$
\left\|\partial(\bar{\partial})^{-1}\right\|_{p, \delta+1} \leq M^{1-s} 1^{s}
$$

where

$$
\frac{1}{p}=(1-s) \frac{1}{q}+s \frac{1}{2}
$$

and

$$
1+\delta=(1-s)(1+\beta)
$$

If we choose $s$ so close to one that

$$
\varepsilon M^{1-s}<1,
$$

then the Neumann series for $\left(I-\mu_{\gamma} \partial(\bar{\partial})^{-1}\right)^{-1}$ converges and (2.19) follows.

Lemma 2.5. Let $p$ and $\delta$ satisfy (2.14), then there exist unique $L_{\delta}^{p}$ solutions to

$$
\left(\bar{\partial}-\mu_{\gamma} \partial\right) u_{1}=v_{1} \in L_{\delta+1}^{p}
$$

and

$$
\left(\bar{\partial}-\partial \mu_{\gamma}\right) u_{2}=v_{2} \in L_{\delta+1}^{p}
$$


Furthermore,

$$
\left\|u_{i}\right\|_{p, \delta}+\left\|D u_{i}\right\|_{p, \delta+1} \leq \frac{2}{\varepsilon} K(p, \delta)\left\|v_{i}\right\|_{p, \delta+1} \quad ; i=1,2
$$

and, for $p>2$,

$$
\left|u_{i}(z)\right| \leq \frac{2}{\varepsilon} K(p, \delta)\left\|v_{i}\right\|_{p, \delta+1}|z|^{-\frac{2}{p}-\delta} .
$$

Proof. We write (2.20) and (2.21) as

$$
\left(I-\mu_{\gamma} \partial(\bar{\partial})^{-1}\right) \bar{\partial} u_{1}=v_{1}
$$

$$
\bar{\partial}\left(I-(\bar{\partial})^{-1} \partial \mu_{\gamma}\right) u_{2}=v_{2}
$$

For $\left(2.20^{\prime}\right)$, apply Lemma 2.4 and then Lemma 2.3. For $\left(2.21^{\prime}\right)$, first apply lemma 2.3 to invert $\bar{\partial}$ and then note that

$$
\left(I-(\bar{\partial})^{-1} \partial \mu_{\gamma}\right)=\left(I-\bar{\mu}_{\gamma} \bar{\partial} \partial^{-1}\right)^{*}
$$

so that the invertibility of $\left(I-(\bar{\partial})^{-1} \partial \mu_{\gamma}\right)$ follows from that of $\left(I-\bar{\mu}_{\gamma} \bar{\partial} \partial^{-1}\right)$, which follows from Lemma 2.4. We leave it to the reader to check that condition (2.18) is commensurate with the duality argument above.

Proof of Proposition 2.1 From Lemma 2.2, we see that solving (2.4) is equivalent to solving (2.8) which is in turn equivalent to finding $f$ satisfying

$$
\left(\bar{\partial}-\mu_{\gamma} \partial\right) f=\mu_{\gamma}
$$

so that Lemma 2.5 guarantees the existence of a unique $f$ such that $F=z+f$ satisfies (2.4). Differentiating (2.24) and applying (2.22) successively yields

$$
\sum_{1 \leq|\alpha| \leq k+1}\left\|D^{\alpha} f\right\|_{p, \delta+1} \leq \frac{2}{\varepsilon} K\left(\sum_{|\alpha| \leq k}\left\|D^{\alpha} \mu_{\gamma}\right\|_{p, \delta+1}\right)\left(1+\sum_{|\alpha| \leq k}\left\|D^{\alpha} \mu_{\gamma}\right\|_{p, \delta+1}\right)
$$

which guarantees (2.5) and (2.6); (2.3) and (2.2) guarantee that the right hand side is finite for $k \leq 2$ (resp.3). Hence $F$ has 3 (resp. 4) derivatives in $L_{l o c}^{p}$ for some $p \geq 2$ and therefore belongs to $C^{2}$ (resp. $C^{3}$ ). 
$F$ tends to the identity as $z$ approaches infinity, hence it is a proper mapping and its degree is equal to one (the degree is the sum of the number of preimages of a generic point, counted with a plus or minus sign depending on whether the determinant is positive or negative at that particular preimage). Once we show that $\operatorname{det}(D F) \neq 0$, it will follow from the fact that $F$ has degree one that $F$ is in fact a global diffeomorphism. To see this, it is enough to check that $F_{z} \neq 0$, because

$$
\operatorname{det}(D F)=\left|F_{z}\right|^{2}-\left|F_{\bar{z}}\right|^{2}=\left(1-\left|\mu_{\gamma}\right|^{2}\right)\left|F_{z}\right|^{2} .
$$

Now, $F_{z}$ satisfies

$$
\left(\bar{\partial}-\partial \mu_{\gamma}\right) G=0
$$

and it is the unique solution of $(2.26)$ of the form

$$
G=1+g ; g \in L_{\delta}^{p}
$$

because (2.26) is equivalent to

$$
\left(\bar{\partial}-\partial \mu_{\gamma}\right) g=\left(\mu_{\gamma}\right)_{z}
$$

and the $L_{\delta}^{p}$ solution to (2.27) was shown to be unique in Lemma 2.5.

On the other hand, we may seek to solve (2.26) by looking for

$$
G=e^{\sigma}
$$

where $\sigma$ must satisfy

$$
\left(\bar{\partial}-\mu_{\gamma} \partial\right) \sigma=\left(\mu_{\gamma}\right)_{z}
$$

so that there exists a unique $\sigma \in L_{\delta}^{p}$ (Lemma 2.5 again) which approaches zero as $|z|$ approaches $\infty$ by (2.23). Hence,

$$
g=G-1=e^{\sigma}-1 \in L_{\delta}^{p}
$$

and we may conclude that

$$
F_{z}=e^{\sigma} \neq 0
$$

so that $F$ is a diffeomorphism .

We now know that $F^{-1}=z+h$ exists; from

$$
F^{-1} \circ F(z)=z
$$


it follows that

$$
h(z+f)=-f(z) \in L_{\delta}^{p}
$$

and then by change of variables $\left(D f_{z} \in L_{\delta}^{p}, p>2\right.$ implies that $f_{z}$ is locally bounded and (2.23) can be used to show that $f_{z}$ approaches zero as $\left.|z| \rightarrow \infty\right)$ it follows that $h \in L_{\delta}^{p}$. Differentiation of (2.28) will show that $h$ satisfies (2.5) and (2.6).

Proof of Proposition 1.3 We begin by extending $\gamma$ to be a $C^{3}$ conductivity on all of $\mathbb{R}^{2}$ satisfying (2.3). We then take $\Phi$ to be the diffeomorphism $F$ whose existence is asserted in proposition 2.1 and let $\Omega^{\prime}$ be the image $F(\Omega)$. The estimate (1.13) is a consequence of (2.6) and the Sobolev inequalities ( $F$ has four derivatives in $L_{l o c}^{p}$ for some $p>2$ ).

Our next order of business is to produce special solutions to (0.6) in all of $\mathbb{R}^{2}$. We shall make use of the following proposition, which is proposition 1.2 in [SU I].

Proposition 2.6. Let $1<p<\infty,-\frac{2}{p}<\delta<1-\frac{2}{p}$. There exist constants $M(p, \delta)$, $N(p, \delta)>0$ such that if $k \in \mathbb{C}$ and $\gamma$ is a $C^{1,1}$ isotropic conductivity satisfying

$$
\begin{gathered}
q=\frac{\Delta(\operatorname{det} \gamma)^{1 / 4}}{(\operatorname{det} \gamma)^{1 / 4}} \in L_{\delta}^{p} \\
\left\|\left(1+|z|^{2}\right)^{1 / 2} q\right\|_{L^{\infty}} \leq \frac{M}{|k|}
\end{gathered}
$$

then there exists a unique solution to

$$
d \gamma d u=0
$$

such that

$$
u=e^{k z}(\operatorname{det} \gamma)^{-1 / 4}(1+r(z, k))
$$

$$
r(\cdot, k) \in L_{\delta}^{p}\left(\mathbb{R}^{2}\right)
$$

Furthermore,

$$
\|r\|_{p, \delta} \leq \frac{N}{|k|}\|q\|_{p, \delta+1}
$$


and

$$
\|D r\|_{p, \delta} \leq N\|q\|_{p, \delta+1}
$$

The last thing we shall do in this section is to combine proposition 2.6 with proposition 2.1 to produce solutions to (2.31) without the isotropy hypothesis. We shall refer to $F_{\gamma}(z)$, the unique mapping (isothermal coordinates) produced in Proposition 2.1. We shall prove

Proposition 2.7. Let $(p, \delta)$ satisfy (2.18). There exists $M(p, \delta)>0$ such that if $k \in \mathbb{C}$ and $\gamma$ is a $C^{1,1}$ conductivity on $\mathbb{R}^{2}$ satisfying

$$
q=\frac{\Delta\left(\operatorname{det} \gamma \circ F_{\gamma}\right)^{1 / 4}}{\left(\operatorname{det} \gamma \circ F_{\gamma}\right)^{1 / 4}} \in L_{\delta}^{p}
$$

and

$$
\left\|\left(1+|z|^{2}\right)^{1 / 2} q\right\|_{L^{\infty}} \leq \frac{M}{|k|}
$$

then there exists a unique solution to

$$
d \gamma d u=0
$$

such that

$$
u=e^{k z}(\operatorname{det} \gamma)^{-1 / 4}(1+\rho(z, k))
$$

and

$$
\rho(\cdot, k) \in L_{\delta}^{p}, D \rho(\cdot, k) \in L_{\delta+1}^{p} .
$$

Furthermore, with uniform convergence on bounded sets,

$$
\lim _{|k| \rightarrow \infty} \frac{\log u(z, k)}{k}=F_{\gamma}(z)
$$

Proof. We first transform (2.37) to the isotropic equation

$$
d F_{\gamma_{*}} \gamma d w=0
$$


Proposition 2.6 implies the existence of a unique $w$ satisfying (2.41) of the form

$$
w=e^{k z}\left(\operatorname{det} \gamma \circ F_{\gamma}^{-1}\right)^{-1 / 4}(1+r(z, k)) .
$$

Now, a solution to (2.37) will be

$$
\begin{aligned}
u=w \circ F_{\gamma} & =e^{k F_{\gamma}(z)}(\operatorname{det} \gamma)^{-1 / 4}\left(1+r\left(F_{\gamma}(z), k\right)\right. \\
& =e^{k z}(\operatorname{det} \gamma)^{-1 / 4}\left(1+r\left(F_{\gamma}(z), k\right) e^{k f(z)} .\right.
\end{aligned}
$$

We previously established, using (2.22) and (2.23), that $f$ and $f_{z}$ were uniformly bounded and approached zero as $|z|$ approached infinity. Hence we may conclude that

$$
r\left(F_{\gamma}(z), k\right) \in L_{\delta}^{p}
$$

and

$$
\left|e^{k f(z)}-1\right| \in L_{\delta}^{p}
$$

and hence that

$$
\rho(z, k)=\left(1+r\left(F_{\gamma}(z), k\right)\right) e^{k f(z)}-1 \in L_{\delta}^{p}
$$

so that we have proved existence.

To see uniqueness, we suppose $u$ solving (2.37) has the form (2.38), then

$$
\begin{aligned}
w & =u \circ F_{\gamma}^{-1} \\
& =e^{F_{\gamma}^{-1}(z) k}\left(\operatorname{det} \gamma \circ F_{\gamma}^{-1}\right)^{-1 / 4}\left(1+\rho\left(F_{\gamma}^{-1}(z), k\right)\right) \\
& =e^{z k}\left(\operatorname{det} \gamma \circ F_{\gamma}^{-1}\right)^{-1 / 4}\left(1+\rho\left(F_{\gamma}^{-1}(z), k\right)\right) e^{k h(z)}
\end{aligned}
$$

and $w$ solves the isotropic equation (2.41).

By mimicking the argument which we just used to establish existence, we see that $w$ has the required form $(2.32)$ (i.e. $\left.\left(1+\rho\left(F_{\gamma}^{-1}(z), h\right)\right) e^{k h(z)}-1 \in L_{\delta}^{p}\right)$. Hence proposition 2.6 implies that $w$ is unique; therefore $u$ is also unique.

It remains only to establish (2.40); we begin with (2.42) which gives

$$
\frac{\log u(z, k)}{k}=F_{\gamma}(z)-\frac{1}{4 k} \log (\operatorname{det} \gamma)+\frac{1}{k} \log \left(1+r\left(F_{\gamma}(z), k\right)\right) .
$$

Now $F_{\gamma}$ is $C^{1,1}$ and $r(\cdot, k)$ and $\operatorname{Dr}(\cdot, k)$ satisfy (2.33) and (2.34) which allows us to conclude that

$$
\begin{aligned}
\|r(\cdot, k)\|_{W_{p}^{s}\left(B_{R}\right)} & \leq\|r\|_{L^{p}\left(B_{R}\right)}^{1-s}\|D r\|_{L^{p}\left(B_{R}\right)}^{s} \widetilde{K}(p, \delta, R) \\
& \leq\left(\frac{1}{k}\right)^{1-s}\|q\|_{L_{\delta+1}^{p}} \widetilde{K}(p, \delta, R)
\end{aligned}
$$


where $W_{p}^{s}\left(B_{R}\right)$ is the fractional order Sobolev space in the ball of radius $R$ and $\delta$ is as in proposition 2.1. The embedding theorem implies that, for $\frac{2}{p}<s<1$, we have

$$
\sup _{z \in B_{R}}|r(z, k)| \leq C\|r(\cdot, k)\|_{W_{p}^{s}\left(B_{R}\right)}
$$

hence $r$, and also $r \circ F_{\gamma}$, approaches zero (uniformly in $|z| \leq R$ ) so that we may deduce (2.40) from (2.43). 
$\S 3$.

In this section we prove Proposition 1.1. We shall assume throughout this section that $\Omega$ is connected. Proposition 1.1 for arbitrary $\Omega$ follows immediately from this special case. We shall denote the components of $\partial \Omega$ by $(\partial \Omega)_{j} ;(\partial \Omega)_{m}$ represents the outer component (i.e., the boundary of the unbounded component of $\mathbb{R}^{2} \backslash \Omega$ ). The proposition will be a corollary of proposition 2.7 and the following two propositions:

Proposition 3.1. Let $\gamma_{1}$ and $\gamma_{2}$ belong to $C^{k}\left(\mathbb{R}^{2}\right)$ and suppose that both are equal to the euclidean conductivity outside an $\epsilon$ neighborhood of $\Omega$. Let

$$
\phi: \partial \Omega \rightarrow \partial \Omega
$$

be an orientation preserving diffeomorphism which maps $(\partial \Omega)_{m}$ to itself. Then $\phi$ can be extended to a diffeomorphism

$$
\Phi: \mathbb{R}^{2} \backslash \Omega \rightarrow \mathbb{R}^{2} \backslash \Omega
$$

such that, for $|\alpha| \leq k$

$$
\left.D^{\alpha}\left(\Phi_{*} \gamma_{1}-\frac{\operatorname{det} \gamma_{1} \circ \Phi^{-1}}{\operatorname{det} \gamma_{2}} \gamma_{2}\right)\right|_{\partial \Omega}=0
$$

and, for $|x|$ sufficiently large

$$
\Phi(x)=x
$$

Proposition 3.2. Suppose that $\phi$ is a diffeomorphism of $\partial \Omega$ which maps $(\partial \Omega)_{m}$ to itself, and such that

$$
\phi_{*} \Lambda_{\gamma_{1}}=\Lambda_{\gamma_{2}}+R
$$

where $R$ is a smoothing operator ${ }^{1}$. Then $\phi$ is orientation preserving, and, if $\Phi$ is the extension of $\phi$ in proposition 3.1, we have, for $|\alpha| \leq 1$,

$$
\left.D^{\alpha}\left(\operatorname{det} \gamma_{1} \circ \Phi^{-1}-\operatorname{det} \gamma_{2}\right)\right|_{\partial \Omega}=0
$$

Remark. Although we do not carry the proof out here, (3.4) is valid for $|\alpha| \leq k$ if $\gamma_{1}$ and $\gamma_{2}$ belong to $C^{k}$. To see this would require carrying out the computation in lemma

1 A smoothing operator maps $L^{2}$ to $C^{\infty}$. For our purposes, the definition in (3.14) below will suffice. 
3.7 below to order $k$. A form of this computation has been carried out in both [KV I] and in [SU III].

In the proof of proposition 1.1, we shall use the following immediate consequence of the two preceding propositions.

Corollary 3.3. Let $\phi$ satisfy (3.3) and map $(\partial \Omega)_{m}$ to itself. Let $\Phi$ be as in proposition 3.1 , then the conductivity

$$
\gamma_{12}= \begin{cases}\gamma_{2} & \text { on } \Omega \\ \Phi_{*} \gamma_{1} & \text { on } \mathbb{R}^{2} \backslash \Omega\end{cases}
$$

is a $C^{1,1}$ conductivity on $\mathbb{R}^{2}$.

Proof of Proposition 1.1 Our first step is to extend $\gamma_{1}$ and $\gamma_{2}$ to be $C^{3}$ conductivities on all of $\mathbb{R}^{2}$, which are equal to the euclidean conductivity outside an $\epsilon$ neighborhood of $\Omega$. Suppose first that $\phi$ maps $(\partial \Omega)_{m}$ to itself and let $\Phi$ be the extension of $\phi$ to $\mathbb{R}^{2} \backslash \Omega$ produced in proposition 3.1 . Let $v$ be the solution to the Dirichlet problem

$$
\begin{gathered}
d \gamma_{2} d v=0 \text { in } \Omega \\
\left.v\right|_{\partial \Omega}=\left.u_{\gamma_{1}}\right|_{\partial \Omega} \circ \phi^{-1}=\left.u_{\gamma_{1}} \circ \Phi^{-1}\right|_{\partial \Omega}
\end{gathered}
$$

where $u_{\gamma_{1}}$ is the special solution to (2.37), with $\gamma=\gamma_{1}$, of the form (2.38). We define

$$
w= \begin{cases}v & \text { for } z \in \Omega \\ u_{\gamma_{1}} \circ \Phi^{-1} & \text { for } z \in \mathbb{R}^{2} \backslash \Omega .\end{cases}
$$

We claim that $w$ is the unique solution $u_{\gamma_{12}}$ to (2.37) of the form (2.38), with $\gamma=\gamma_{12}$, as defined in (3.5). If we recall (1.1) we see that $w$ satisfies (2.37) in $\mathbb{R}^{2} \backslash \Omega$; it has the form (2.38) because $u_{\gamma_{1}}$ does and because $\Phi$ is the identity outside a large ball. Equation (3.6) shows that $w$ satisfies the differential equation in $\Omega$ and (3.7) implies continuity across $\partial \Omega$. The point that we must check, then, is the continuity of $\gamma_{12} d w$ across $\partial \Omega$.

$$
\text { In } \mathbb{R}^{2} \backslash \Omega \text {, }
$$

$$
\gamma_{12} d w=\Phi_{*} \gamma_{1} d\left(u_{\gamma_{1}} \circ \Phi^{-1}\right)
$$

As we approach $\partial \Omega$ from $\mathbb{R}^{2} \backslash \Omega$, (1.1) and (3.8) imply that

$$
\begin{aligned}
\gamma_{12} d w & \rightarrow \Lambda_{\Phi_{*} \gamma_{1}}\left(\left.u_{\gamma_{1}} \circ \Phi^{-1}\right|_{\partial \Omega}\right) \\
& =\phi_{*} \Lambda_{\gamma_{1}}\left(\left.u_{\gamma_{1}} \circ \Phi^{-1}\right|_{\partial \Omega}\right) \\
& =\Lambda_{\gamma_{2}}\left(\left.u_{\gamma_{1}} \circ \Phi^{-1}\right|_{\partial \Omega}\right)
\end{aligned}
$$


which, according to (3.7) and (3.8), is exactly the limit of $\gamma_{12} d w$ as we approach $\partial \Omega$ from inside $\Omega$.This establishes our claim.

It follows from (2.40) and (3.8) that $\left.F_{\gamma_{12}}\right|_{\Omega}$ is a $\left(\gamma_{2}-e\right)$ conformal map and from (3.7) that

$$
\left.F_{\gamma_{12}}\right|_{\partial \Omega}=\left.F_{\gamma_{1}}\right|_{\partial \Omega} \circ \phi^{-1} .
$$

Hence $F_{\gamma_{12}}^{-1} \circ F_{\gamma_{1}}$ is $\left(\gamma_{1}-\gamma_{2}\right)$ conformal and

$$
\left.F_{\gamma_{12}}^{-1} \circ F_{\gamma_{1}}\right|_{\partial \Omega}=\phi .
$$

This establishes (1.8) with $F_{\gamma_{12}}^{-1} \circ F_{\gamma_{1}}$ in place of $\Phi$. The estimate (1.9) follows from similiar estimates on $F_{\gamma_{12}}^{-1}$ and $F_{\gamma_{1}}$. The latter is a consequence of (1.13). The former does not follow directly from (1.13) because we have not verified that $\gamma_{12}$ belongs to $C^{3}\left(\mathbb{R}^{2}\right)$ (see the remark following proposition 3.2); however, $F_{\gamma_{12}}^{-1}$ solves the elliptic boundary value problem (1.27) in $\Omega$ with $C^{3}$ coefficients and $C^{3}$ boundary data $\phi$, hence $F_{\gamma_{12}}^{-1}$ must belong to $C^{3}$ and satisfy an estimate of the form (1.9).

Finally, suppose that $\phi$ does not map $(\partial \Omega)_{m}$ to itself; i.e., for some $j \neq m$

$$
\phi:(\partial \Omega)_{j} \rightarrow(\partial \Omega)_{m}
$$

Let $F$ be a diffeomorphism from $\Omega$ to itself which maps $(\partial \Omega)_{m}$ to $(\partial \Omega)_{j}$. To see that such an $F$ exists, proceed as follows: map $\Omega$ diffeomorphically to a domain $\widetilde{\Omega}$ whose boundary consists of circles (the existence of such a diffeomorphism is guaranteed by the Riemann mapping theorem for multiply connected domains: we leave the radii of the circles and their locations to be determined so that the mapping will exist), use a fractional linear transformation to map the outer bounding circle to an inner circle, and finally scale the image circles and move them about with the aid of flows induced by vector fields to force the image to again be $\widetilde{\Omega}$.

If $f$ denotes the restriction of $F$ to $\partial \Omega$,we have

$$
(\phi \circ f)_{*} \Lambda_{F_{*}^{-1} \gamma_{1}}=\Lambda_{\gamma_{2}}
$$

and $(\phi \circ f)$ maps $(\partial \Omega)_{m}$ to itself so that $(\phi \circ f)$ extends to a $F_{*}^{-1} \gamma_{1}-\gamma_{2}$ conformal map. Composition with $F$ now yields the desired extension of $\phi$.

Proof of Proposition 3.1 The proof of proposition 3.1 breaks into two parts. The first is to find a $\Phi$ satisfying (3.1) in a neighborhood of $\partial \Omega$. This is equivalent to formally solving the Cauchy problem (1.27) with Cauchy data $\left.\Phi\right|_{\partial \Omega}=\phi$. This can always be accomplished because (1.27) is elliptic. The second part is to prove: 
Lemma 3.4. Any orientation preserving diffeomorphism mapping a neighborhood of $\partial \Omega$ to a neighborhood of $\partial \Omega$ can be extended, from a possibly smaller neighborhood, to a diffeomorphism $\Phi$ of $\mathbb{R}^{2}$. If $\tilde{\Phi}$ maps a neighborhood of $\left(\partial \Omega_{m}\right)$ to itself, then $\Phi$ may be chosen to satisfy (3.2).

Proof Let $\mathbf{D}$ denote the unit disk. We shall prove below:

Lemma 3.5. Any orientation preserving diffeomorphism mapping a neighborhood of $\partial \mathbf{D}$ to a neighborhood of $\partial \mathbf{D}$ can be extended, from a possibly smaller neighborhood, to a diffeomorphism of $\mathbb{R}^{2}$ which equals the identity outside an $\epsilon$ neighborhood of $\mathbf{D}$.

We contend that, with the aid of lemma 3.5, we can produce an orientation preserving diffeomorphism $N$ from $\mathbb{R}^{2}$ to itself which maps $\Omega$ onto a domain $\widetilde{\Omega}$ whose boundary consists only of circles. The first step is to let $S$ be a diffeomorphism of $\Omega$ onto $\widetilde{\Omega}-$ the existence of such a mapping is guaranteed by the Riemann mapping theorem for multiply connected domains. Next, let $R_{j}$ denote a diffeomorphism from $\left(\mathbb{R}^{2} \backslash \Omega\right)_{j}$, the $j^{\text {th }}$ component of $\mathbb{R}^{2} \backslash \Omega$, (this is simply connected because we have assumed that $\Omega$ is connected) onto the $j^{t h}$ component of $\mathbb{R}^{2} \backslash \widetilde{\Omega}$ (the existence of such a mapping is guaranteed by the Riemann mapping theorem). Both diffeomorphisms are smooth up to the boundary and extend to a full neighborhood of their common boundary. As a consequence of lemma 3.5 , we may extend the diffeomorphism $S \circ R_{j}^{-1}$ (maps a neighborhood of a circle to a neighborhood of a circle) to a diffeomorphism $\Psi_{j}$ of $\mathbb{R}^{2}$ which is the identity, except in a neighborhood of the boundary circle. We may now define $N$ by

$$
N(x)=\left\{\begin{array}{ll}
S & \text { for } x \in \Omega \\
\Psi_{j} & \text { for } x \in\left(\mathbb{R}^{2} \backslash \Omega\right)_{j}
\end{array} .\right.
$$

It now suffices to prove lemma 3.4 for $\widetilde{\Omega}$; conjugation with $N$ then provides the extension for $\Omega$.

To prove the lemma for $\widetilde{\Omega}$, we note that each connected component $\left(\mathbb{R}^{2} \backslash \widetilde{\Omega}\right)_{j}$ is a disk and that our diffeomorphism $\tilde{\Phi}$ maps $(\partial \widetilde{\Omega})_{j}$ to $(\partial \widetilde{\Omega})_{k}$ for some $j, k \neq m$. Lemma 3.5 guarantees that $\tilde{\Phi}$ extends inside each disk (scale first so that both disks have radius one). If $j=k=m$, we again employ lemma 3.5 to extend $\tilde{\Phi}$ outside this disk. This completes the proof of the lemma.

All that remains in the proof of proposition 3.1 is the

Proof of lemma 3.5 In polar coordinates, an orientation preserving diffeomorphism of $\mathbf{D}$ is given by:

$$
\Psi(r, \theta)=\left(\begin{array}{c}
1 \\
\phi(\theta)
\end{array}\right)+(r-1)\left(\begin{array}{c}
\tilde{a}_{1}(\theta) \\
\tilde{a}_{2}(\theta)
\end{array}\right)+(r-1)^{2} \widetilde{R}(r, \theta)
$$


where

$$
\phi^{\prime}(\theta)>\epsilon>0
$$

and

$$
\tilde{a}_{1}(\theta)>\epsilon>0
$$

We shall choose

$$
A(r, \theta)=\left(\begin{array}{c}
r \\
\theta+\mu(r)(\phi(\theta)-\theta)
\end{array}\right)
$$

and

$$
B(r, \theta)=\left(\begin{array}{c}
r \\
\theta
\end{array}\right)+(r-1) \mu(r)\left(\left(\begin{array}{c}
a_{1}-1 \\
a_{2}
\end{array}\right)+(r-1) R\right)
$$

We claim that $A$ and $B$ are diffeomorphisms whose composition, $B \circ A$, is the desired extension of $\Psi$. In (3.11), $a_{1}, a_{2}$, and $R$ represent the composition of $\tilde{a}_{1}, \tilde{a}_{2}$, and $\tilde{R}$ with $\phi^{-1}(\theta)$. The function $\mu(r)$ is a cutoff, which is identically equal to one in some neighborhood of $\{r=1\}$ and identically equal to zero outside some other neighborhood. This is enough, along with (3.9), to check that $A$ is a local diffeomorphism. To guarantee that $B$ is a local diffeomorphism we shall require that

$$
\begin{array}{ll}
\mu(r)=1 & \text { for } \quad|r-1|<\delta \\
\mu(r)=0 & \text { for } \quad|r-1|>M \delta
\end{array}
$$

and that

$$
0>(r-1) \mu^{\prime}(r)>-\frac{\epsilon}{2}
$$

It is possible to find such a $\mu$ (with $M=e^{2 / \epsilon}$ and $\delta$ arbitrarily small) because

$$
\int_{\delta+1}^{M \delta+1} \frac{1}{r-1} d r=\log M
$$

Now,

$$
D B(r, \theta)=\left(\begin{array}{cc}
(1-\mu)+\mu a_{1}+\mu^{\prime}(r-1)\left(a_{1}-1\right) & (r-1) \mu a_{1}^{\prime} \\
(r-1) \mu^{\prime} a_{2}+\mu a_{2} & 1+(r-1) \mu a_{2}
\end{array}\right)+(r-1) \widetilde{B}(r, \theta)
$$

It follows from (3.12) and (3.10) that, for a sufficiently small choice of $\delta$, the diagonal entries of $D B$ are bounded from below and the off diagonal entries are small enough to 
guarantee the invertibility of $D B$. Because both $A$ and $B$ are equal to the identity off a compact set, it follows from degree arguments that both are global diffeomorphisms.

It remains only to prove proposition 3.2. As in the proof of proposition 3.1, we begin with a special case.

Lemma 3.6. Proposition 3.2 holds in the case that $\gamma_{1}$ and $\gamma_{2}$ are isotropic and $\Omega$ is a disk.

Proof In polar coordinates, an isotropic $\gamma$ is given by

$$
\gamma d u=\alpha r \frac{\partial u}{\partial r} d \theta-\frac{\alpha}{r} \frac{\partial u}{\partial \theta} d r \quad ; \alpha=(\operatorname{det} \gamma)^{1 / 2}
$$

and

$$
\left.\gamma d u\right|_{r=1}=\left.\alpha \frac{\partial u}{\partial r}\right|_{r=1} d \theta
$$

To establish lemma 3.6 we shall need the following description of the operator $\Lambda_{\gamma}$ :

\section{Lemma 3.7.}

$$
\left.\Lambda_{\gamma} f=\left(\left.\alpha\right|_{r=1}\left(\left|\frac{\partial}{\partial \theta}\right|\right) f\right)+\left.\frac{1}{2} \frac{\partial \alpha}{\partial r}\right|_{r=1} f-\left.\frac{i}{2} \frac{\partial \alpha}{\partial \theta}\right|_{r=1} \operatorname{sgn}\left(\frac{\partial}{\partial \theta}\right) f+R f\right) d \theta
$$

where

$$
\begin{gathered}
\left|\frac{\partial}{\partial \theta}\right|: e^{i n \theta} \mapsto|n| e^{i n \theta} \\
\operatorname{sgn} \frac{\partial}{\partial \theta}: e^{i n \theta} \mapsto \operatorname{sgn}(n) e^{i n \theta}
\end{gathered}
$$

and

$$
R: H^{1 / 2}\left(S^{1}\right) \mapsto H^{3 / 2}\left(S^{1}\right) \quad\left(H^{s}=W^{s, 2}\right)
$$

Proof. There are many routes to (3.13). The most general is to note that $\Lambda_{\gamma}$ is a pseudodifferential operator and to compute the first two terms in its symbol expansion. Because we are in $D$, the disk in two dimensions, we can give a direct proof which avoids any extra machinery.

Let $u_{0}$ solve

$$
\begin{gathered}
\Delta u_{0}=0 \\
\left.u_{0}\right|_{r=1}=f \in H^{1 / 2}\left(S^{1}\right)
\end{gathered}
$$


then $u_{0} \in H^{1}(D)$. Let $u_{1}$ solve

$$
\begin{gathered}
\Delta u_{1}=-\frac{\partial u_{0}}{\partial r} \\
\left.u_{1}\right|_{r=1}=0
\end{gathered}
$$

and $u_{2}$ solve

$$
\begin{gathered}
\Delta u_{2}=\frac{-1}{r} \frac{\partial u_{0}}{\partial \theta} \\
\left.u_{2}\right|_{r=1}=0
\end{gathered}
$$

so that $u_{1}$ and $u_{2} \in H^{2}(D)$. If $u$ solves

$$
\begin{gathered}
d(\gamma d u)=\left(\alpha \Delta u+\alpha_{r} \frac{\partial u}{\partial r}+\frac{\alpha_{\theta}}{r^{2}} \frac{\partial u}{\partial \theta}\right) r d r_{\wedge} d \theta=0 \\
\left.u\right|_{\partial \Omega}=f
\end{gathered}
$$

and we set

$$
u_{R}=u-u_{0}-\frac{\alpha_{r}}{\alpha} u_{1}-\frac{\alpha_{\theta}}{r \alpha} u_{2}
$$

then $u_{R}$ satisfies

$$
\begin{gathered}
\alpha \Delta u_{R}+\alpha_{r} \frac{\partial u_{R}}{\partial r}+\frac{\alpha_{\theta}}{r^{2}} \frac{\partial u_{R}}{\partial \theta}=A u_{1}+B u_{2} \\
\left.u_{R}\right|_{r=1}=0
\end{gathered}
$$

where $A$ and $B$ are first order differential operators with coefficients which are smooth away from the origin, so that $u_{R} \in H_{l o c}^{3}(D \backslash\{0\})$ and the mapping

$$
\left.f \stackrel{\widetilde{R}}{\mapsto} \alpha \frac{\partial u_{R}}{\partial r}\right|_{r=1}
$$

satisfies (3.14).

If we take $f=e^{i n \theta}$, however, we may compute that

$$
\begin{gathered}
u_{0}=r^{|n|} e^{i n \theta} \\
u_{1}=\frac{-|n|}{(2|n|+1)}\left(r^{|n|+1}-r^{|n|}\right) e^{i n \theta} \\
u_{2}=\frac{-i n}{(2|n|+1)}\left(r^{|n|+1}-r^{|n|}\right) e^{i n \theta}
\end{gathered}
$$

Hence,

$$
\begin{aligned}
\left.\gamma d u\right|_{r=1} & =\left.\alpha \frac{\partial u}{\partial r}\right|_{r=1} d \theta \\
& =\left(\alpha|n|-\frac{\alpha_{r}}{2}-\frac{i \alpha_{\theta}}{2} \operatorname{sgn}(n)+g\left(n, \alpha, \alpha_{r}, \alpha_{\theta}, \Delta \alpha\right)\right) e^{i n \theta} d \theta
\end{aligned}
$$


where $\mathrm{g}$ is $O\left(\frac{1}{|n|}\right)$. Finally, if we define

$$
R e^{i n \theta}=\widetilde{R} e^{i n \theta}+g\left(n, \alpha, \alpha_{r}, \alpha_{\theta}, \Delta \alpha\right) e^{i n \theta}
$$

we obtain (3.13).

A consequence of (3.13) and (3.3) is

$$
\phi_{*}\left(\left.\alpha_{1}\right|_{r=1}\left|\frac{\partial}{\partial \theta}\right| \otimes d \theta\right)=\left.\alpha_{2}\right|_{r=1}\left|\frac{\partial}{\partial \theta}\right| \otimes d \theta
$$

and that

$$
\phi_{*}\left(\left(\left.\frac{\partial \alpha_{1}}{\partial r}\right|_{r=1}-\left.i \frac{\partial \alpha_{1}}{\partial \theta}\right|_{r=1} \operatorname{sgn} \frac{\partial}{\partial \theta}\right) \otimes d \theta\right)=\left(\left.\frac{\partial \alpha_{2}}{\partial r}\right|_{r=1}-\left.i \frac{\partial \alpha_{2}}{\partial \theta}\right|_{r=1} \operatorname{sgn} \frac{\partial}{\partial \theta}\right) \otimes d \theta .
$$

Computing the push forward in (3.15) and (3.16) yields

$$
\operatorname{sgn}\left(\phi^{-1^{\prime}}(\theta)\right) \alpha_{1}\left(1, \phi^{-1}(\theta)\right)=\alpha_{2}(1, \theta)
$$

and

$$
\begin{aligned}
\frac{\partial \alpha_{1}}{\partial r}\left(1, \phi^{-1}(\theta)\right) \phi^{-1^{\prime}}(\theta) & =\frac{\partial \alpha_{2}}{\partial r}(1, \theta) \\
\frac{\partial \alpha_{1}}{\partial \theta}\left(1, \phi^{-1}(\theta)\right) \phi^{-1^{\prime}}(\theta) & =\frac{\partial \alpha_{2}}{\partial \theta}(1, \theta) .
\end{aligned}
$$

Since both $\alpha_{1}$ and $\alpha_{2}$ are positive, $\phi^{-1^{\prime}}$ must be positive so that $\phi$ is orientation preserving. To see that $\left(3.15^{\prime}\right),\left(3.16^{\prime}\right)$, and $\left(3.16^{\prime \prime}\right)$ establish (3.4), we need only check that, in polar coordinates,

$$
D \Phi(1, \theta)=\phi^{\prime}(1, \theta)\left(\begin{array}{ll}
1 & 0 \\
0 & 1
\end{array}\right)
$$

Recall that $\Phi$ is a formal solution (to order $\mathrm{k}$ ) of (1.27) with Cauchy data $\phi$, and that (1.27), in the case that $\gamma_{1}$ and $\gamma_{2}$ are isotropic, is just the Cauchy Riemann equations. This means that $\Phi$ maps $\partial D$ to itself and is conformal to order $\mathrm{k}$, which implies, in particular, (3.17).

This completes the proof of lemma 3.6.

Proof of Proposition 3.2 Suppose first that $\Omega$ is simply connected. If we let $\Phi_{1}$ and $\Phi_{2}$ denote isothermal coordinates for $\gamma_{1}$ and $\gamma_{2}$ respectively, then $\Phi_{1 *} \gamma_{1}$ and $\Phi_{2 *} \gamma_{2}$ are isotropic conductivities on the domains $\Phi_{1}(\Omega)$ and $\Phi_{2}(\Omega)$ respectively. If we let $\Psi_{i}$ denote 
conformal maps from the $\Phi_{i}(\Omega)$ to the unit disk, $\left(\Psi_{i} \circ \Phi_{i}\right)_{*} \gamma_{i}$ are isotropic conductivities on the disk. Because

$$
\phi_{*} \Lambda_{\gamma_{1}}=\Lambda_{\gamma_{2}}+R
$$

we have

$$
\left.\left(\phi\left(\psi_{1} \phi_{1}\right)^{-1}\right)_{*} \Lambda_{\left(\Psi_{1} \Phi_{1}\right)_{*} \gamma_{1}}=\left(\psi_{2} \phi_{2}\right)^{-1}\right)_{*} \Lambda_{\left(\Psi_{2} \Phi_{2}\right)_{*} \gamma_{2}}+\widetilde{R}
$$

or

$$
\left(\psi_{2} \phi_{2} \phi\left(\psi_{1} \phi_{1}\right)^{-1}\right)_{*} \Lambda_{\left(\Psi_{1} \Phi_{1}\right)_{*} \gamma_{1}}=\Lambda_{\left(\Psi_{2} \Phi_{2}\right)_{*} \gamma_{2}}+\widetilde{\widetilde{R}}
$$

Lemma 3.6 implies that $\left(\psi_{2} \phi_{2} \phi\left(\psi_{1} \phi_{1}\right)^{-1}\right)$ is orientation preserving and

$$
\left.D^{\beta}\left(\operatorname{det}\left(\Psi_{1} \Phi_{1}\right)_{*} \gamma_{1} \circ \widetilde{\Phi}\right)\right|_{\partial D}=\left.D^{\beta}\left(\operatorname{det}\left(\Psi_{2} \Phi_{2}\right)_{*} \gamma_{2}\right)\right|_{\partial D} \quad ; \quad|\beta| \leq 1
$$

where $\widetilde{\Phi}$ is the extension in proposition 3.1 with $\phi$ replaced by $\psi_{2} \phi_{2} \phi\left(\psi_{1} \phi_{1}\right)^{-1}$. Now $\Phi$ is related to $\widetilde{\Phi}$ by

$$
\Phi=\left(\Psi_{2} \Phi_{2}\right)^{-1} \circ \widetilde{\Phi} \circ\left(\Psi_{1} \Phi_{1}\right)
$$

so that (3.18) implies (3.4) and the propositon is true in the case that $\Omega$ is simply connected.

Finally, suppose that $\Omega$ is not simply connected. Let $\widetilde{\Omega}$ denote the complement to $\left(\mathbb{R}^{2} \backslash \Omega\right)_{m}$, the unbounded component of $\mathbb{R}^{2} \backslash \Omega$ (i.e. $\Omega$ with the holes filled in), and suppose that $\gamma_{i}$ has been extended to be a $C^{3}$ conductivity $\tilde{\gamma}_{i}$ on $\widetilde{\Omega}$. We claim that

$$
\Lambda_{\tilde{\gamma}_{i}}=\left(\Lambda_{\gamma_{i}}\right)_{N}+R
$$

where $R$ is a smoothing operator and $\left(\Lambda_{\gamma_{i}}\right)_{N}$ is a restriction of $\Lambda_{\gamma_{i}}$; namely, for $f \in C^{3}(\partial \widetilde{\Omega})$,

$$
\left(\Lambda_{\gamma_{i}}\right)_{N} f=\left.\gamma_{i} d u\right|_{\partial \widetilde{\Omega}}
$$

where $u$ satisfies

$$
\begin{gathered}
d \gamma_{i} d u=0 \text { for } x \in \Omega \\
\left.u\right|_{\partial \widetilde{\Omega}}=f \\
\left.u\right|_{\partial \Omega \backslash \partial \widetilde{\Omega}}=0
\end{gathered}
$$

Our claim follows from the fact that the difference between $u$ and the solution to the Dirichlet problem in $\widetilde{\Omega}$ is smooth in a neighborhood of $\partial \widetilde{\Omega}$. To see this, multiply the difference by a cutoff supported in a neigborhood of $\partial \widetilde{\Omega}$ and apply standard elliptic estimates to this product (see $[\mathrm{KV} \mathrm{I}]$ e.g.).

Hence,the hypothesis (3.3) on $\Omega$ implies the same hypothesis on $\widetilde{\Omega}$, which is simply connected. Thus we may conclude from the simply connected case that $\phi$ is orientation 
preserving and that (3.4) holds on $\partial \widetilde{\Omega}$, which coincides with $(\partial \Omega)_{m}$. However, if we push forward both $\gamma_{1}$ and $\gamma_{2}$ by an inversion in a sphere in any bounded component of $\mathbb{R}^{2} \backslash \Omega$, that boundary component will become the new $(\partial \Omega)_{m}$. Hence $\phi$ is orientation preserving, (3.4) holds on all of $\partial \Omega$, and the proposition in the case of a general $\Omega$ follows from the simply connected case. 


\section{Bibliography}

[A] Ahlfors L., Quasiconformal Mappings, Van Nostrand 1966.

[C] A. P. Calderón, "On an inverse boundary value problem", Seminar on Numerical Analysis and its Applications to Continuum Physics. Soc. Brasileira de Matematica. Rio de Janeiro, 1980.

[CH] Courant-Hilbert, Methods of Mathematical Physics Vol II, Interscience, 1962.

[I] Isakov, V., On the uniqueness of recovery of a discontinuous conductivity coefficient, CPAM (to appear).

[KV I] Kohn, R. and Vogelius, M., Determining conductivity by boundary measurement I, CPAM, 37 (1984), 113-123.

[KV II] Determining conductivity by boundary measurements II Interior results, CPAM, 38, 1985, 643-667.

[KV III $]$, Identification of an unknown conductivity by means of measurements at the boundary, SIAM-AMS Proceeding, Vol 14, 1984.

[L-U] Lee, J. and Uhlmann, G., Determining real analytic anisotropic conductivities by boundary measurements (preprint).

[NW] Nirenberg L. and Walker H., Null spaces of elliptic partial differential operators in $\mathbb{R}^{n}$, J. Math. Anal. Appl. 42, 1973, pp271-301.

[S] Stein, E., Singular Integrals and Differentiability Properties of Functions, Princeton University Press 1970.

[Sp] Spivak, M., A comprehensive Introduction to Differential Geometry, Vol IV, Publish or Perish, 19.

[SU I] J. Sylvester and G. Uhlmann, A uniqueness theorem for an inverse boundary value problem in electrical prospection, Comm. Pure Appl. Math. 39 (1986) 92-112.

[SU II] A global uniqueness theorem for an inverse boundary value problem, Ann. Math. 125 (1987) 153-169.

[SU III] Inverse boundary value problems at the boundary-continuous dependence, Comm. Pure Appl. Math. 41 (1988) 197-219. 